\title{
CD3G or CD3D Knockdown in Mature, but Not Immature, $T$ Lymphocytes Similarly Cripples the Human TCR $\alpha \beta$ Complex
}

\author{
Beatriz Garcillán ${ }^{1 t}$, Patricia Fuentes ${ }^{21}$, Ana V. Marin ${ }^{1+}$, Rebeca F. Megino', \\ Daniel Chacon-Arguedas ${ }^{1}$, Marina S. Mazariegos ${ }^{1}$, Anaïs Jiménez-Reinoso', \\ Miguel Muñoz-Ruiz ${ }^{1}$, Raquel G. Laborda ${ }^{1}$, Paula P. Cárdenas ${ }^{1 *}$, \\ Edgar Fernández-Malavé1*, Maria L. Toribio ${ }^{2}$ and José R. Regueiro ${ }^{\text {** }}$
}

OPEN ACCESS

Edited by:

Andrea Graziani,

University of Turin, Italy

Reviewed by:

Cynthia L. Bristow,

Alpha-1 Biologics, United States

Ana Cuenda,

Consejo Superior de Investigaciones

Cientificas (CSIC), Spain

${ }^{*}$ Correspondence:

Paula P. Cárdenas

paulcard@ucm.es

Edgar Fernández-Malavé

edfernan@med.ucm.es José R. Regueiro regueiro@ucm.es

tThese authors have contributed equally to this work

Specialty section: This article was submitted to

Signaling,

a section of the journa

Frontiers in Cell and Developmental Biology

Received: 20 September 2020 Accepted: 24 May 2021

Published: 25 June 2021

Citation:

Garcillán B, Fuentes P, Marin AV, Megino RF, Chacon-Arguedas $D$, Mazariegos MS, Jiménez-Reinoso A

Muñoz-Ruiz M, Laborda RG, Cárdenas PP, Fernández-Malavé E, Toribio ML and Regueiro JR (2021)

CD3G or CD3D Knockdown in Mature, but Not Immature, T Lymphocytes Similarly Cripples

the Human TCR $\alpha \beta$ Complex. Front. Cell Dev. Biol. 9:608490. doi: 10.3389/fcell.2021.608490
Department of Immunology, Ophthalmology and ENT, Complutense University School of Medicine and 12 de Octubre Health Research Institute (imas12), Madrid, Spain, ${ }^{2}$ Interaction with the Environment Program, Immune System Development and Function Unit, Centro de Biología Molecular Severo Ochoa, CSIC-UAM, Madrid, Spain

The human $\alpha \beta$ T-cell receptor (TCR) is composed of a variable heterodimer (TCR $\alpha \beta$ ) and three invariant dimers (CD3 $\gamma \varepsilon, \mathrm{CD} 3 \delta \varepsilon$, and $\left.\zeta \zeta / C D 247_{2}\right)$. The role of each invariant chain in the stepwise interactions among TCR chains along the assembly is still not fully understood. Despite the high sequence homology between CD3 $\gamma$ and CD3 8 , the clinical consequences of the corresponding immunodeficiencies (ID) in humans are very different (mild and severe, respectively), and mouse models do not recapitulate findings in human ID. To try to understand such disparities, we stably knocked down (KD) CD3D or CD3G expression in the human Jurkat T-cell line and analyzed comparatively their impact on TCR $\alpha \beta$ assembly, transport, and surface expression. The results indicated that TCR ensembles were less stable and CD3 $\varepsilon$ levels were lower when CD3 $\gamma$, rather than CD38, was scarce. However, both defective TCR ensembles were strongly retained in the ER, lacked $\zeta \zeta / C D 2472$, and barely reached the T-cell surface $(<11 \%$ of normal controls) in any of the CD3 KD cells. This is in sharp contrast to human CD3 $\gamma$ ID, whose mature T cells express higher levels of surface TCR (>30\% vs. normal controls). CD3 KD of human T-cell progenitors followed by mouse fetal thymus organ cultures showed high plasticity in emerging immature polyclonal $\mathrm{T}$ lymphocytes that allowed for the expression of significant TCR levels which may then signal for survival in CD3 $\gamma$, but not in CD3 $\delta$ deficiency, and explain the immunological and clinical disparities of such ID cases.

Keywords: CD3G, CD3D, shRNA knockdown, T-cell receptor (TCR), TCR assembly, T-cell progenitors, immunodeficiency

\section{INTRODUCTION}

$\mathrm{T}$ lymphocytes detect the presence of antigens through a cell surface complex termed the T-cell receptor (TCR). This receptor is composed of a variable heterodimer (either TCR $\alpha \beta$ or TCR $\gamma \delta$ ) responsible for ligand recognition and three invariant dimers $\left(\mathrm{CD} 3 \gamma \varepsilon, \mathrm{CD} 3 \delta \varepsilon\right.$, and $\left.\zeta \zeta / C D 247_{2}\right)$ that participate in the assembly and surface expression of the TCR complex and in the delivery of intracellular signals (Call et al., 2004). Binding of the peptide-MHC complex by the TCR results in CD3 conformational changes (Schamel et al., 2019) and the phosphorylation of tyrosine residues within the immunoreceptor tyrosine-based activation motifs (ITAM) of the cytoplasmic tails of the CD3 and $\zeta \zeta / C D 2472$ chains (Love and Hayes, 2010), which eventually lead to the activation of 
several signaling cascades that result in T-cell activation and the induction of a cellular immune response (Brownlie and Zamoyska, 2013).

TCR assembly is an ordered process that begins in the endoplasmic reticulum (ER). CD3 heterodimers are formed through interactions between their extracellular Ig domains and associate with TCR chains through intramembrane contacts to form the hexameric TCR $(\alpha \beta \gamma \varepsilon \delta \varepsilon)$, which is exported to the Golgi apparatus where it interacts with the $\zeta \zeta / C D 247_{2}$ homodimer (Dietrich et al., 1999; Wucherpfennig et al., 2010). The result is an octameric complex that is sorted to the plasma membrane. Correct folding and assembly is required for transport to the cell surface, and misfolded or single subunits and partial receptor complexes are retained in the ER and eventually retro-translocated to the cytosol for ER-associated degradation. Hexameric TCR ensembles that reach the Golgi apparatus but fail to assemble with $\zeta \zeta / C D 247_{2}$ homodimers are sorted to the lysosomal pathway for degradation (Bonifacino et al., 1990; Wileman et al., 1990, 1993; Letourneur and Klausner, 1992; Lee, 1998; Delgado and Alarcon, 2005).

$\mathrm{CD} 3 \gamma$ and $\mathrm{CD} 3 \delta$ proteins are highly homologous, and in some vertebrates, they are a single CD $3 \gamma / \delta$ chain (Göbel and Dangy, 2000). In humans, it has been shown that the intracellular and transmembrane, but not the extracellular, domain of CD3 $\delta$ can substitute those of CD3 $\gamma$ for assembly and expression (Wegener et al., 1995). The functions of the individual CD3 chains for assembly and expression and the stepwise interactions among the TCR subunits are still not fully understood and differ between humans and mice.

Several studies have attempted to address these questions studying knockout (KO) mice [for CD3 $\gamma$ (Haks et al., 1998)

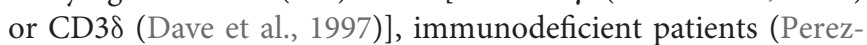
Aciego et al., 1991), mutant Jurkat T-cell lines (Geisler, 1992), or heterologous systems (Kappes and Tonegawa, 1991; Call et al., 2002; Szymczak et al., 2004), but they yielded contradictory results regarding the requirement of $\mathrm{CD} 3 \gamma$ and $\mathrm{CD} 3 \delta$ proteins for surface TCR expression and signaling. Indeed, $\mathrm{KO}$ mice, mutant Jurkat T-cell lines, and reconstituted B-cell microsomes showed that TCR assembly, surface expression, and signaling required $\mathrm{CD} 3 \gamma$ and $\mathrm{CD} 3 \delta$ proteins, whereas immunodeficient patients and reconstituted HeLa or $293 \mathrm{~T}$ cells did not. Here, we knocked down (KD) $\mathrm{CD} 3 \gamma$ and $\mathrm{CD} 3 \delta$ in mature and immature $\alpha \beta \mathrm{T}$ cells to analyze their role in TCR assembly and expression.

\section{MATERIALS AND METHODS}

\section{Cell Lines}

Mycoplasma-free Jurkat E6-1 cells, a clone of the Jurkat JM CD4 ${ }^{+}$ T-cell leukemia cell line established from the peripheral blood of a 14-year-old boy with acute T-cell leukemia (Schneider et al., 1977), was used because they express all TCR chains (Abraham and Weiss, 2004). The CH7C17 Jurkat cell line is a mutant of Jurkat E6-1 cell line, negative for TCR and reconstituted by transfection with a TCR specific for hemagglutinin peptide bearing V $\alpha 1.4$ and V $\beta 3.1$ TCR chains (Hewitt et al., 1992). The Herpesvirus Saimiri (HVS) CD $3 \gamma^{-/-}$cell line was previously generated from peripheral blood lymphocytes (PBL) of a congenital CD3 $\gamma$-deficient individual (Pacheco-Castro et al., 1998). Cells were grown in RPMI 1640 medium (HyClone, Logan, UT, United States) supplemented with $10 \%$ FCS and $2 \mathrm{mM}$ L-glutamine (Life Technologies, Carlsbad, CA, United States); $400 \mu \mathrm{g} / \mathrm{ml}$ hygromycin B (Life Technologies) was supplemented for $\mathrm{CH7C17}$ cell line, and $50 \mathrm{IU} / \mathrm{ml}$ human recombinant human IL-2 (provided by Craig W. Reynolds, Frederick Cancer Research and Development Center, National Cancer Institute, National Institutes of Health, Frederick, MD, United States) was supplemented every 2 days for the HVS CD3 $\gamma^{-/-}$cell line. The HBP-ALL cell line was originally generated from a T-cell acute lymphoblastic leukemia patient. PBL were isolated from healthy donors with informed consent and IRB authorization. During the assay, all cells were maintained in RPMI 1640 medium supplemented with $10 \%$ FCS and $2 \mathrm{mM}$ L-glutamine.

\section{Lentivirus Production, Transduction, and KD}

To generate KD cells, the pLKO.1-CMV-TurboGFP ${ }^{\mathrm{TM}}$ plasmid backbone containing a puromycin selectable marker, a turboGFP marker, and a short hairpin (sh) RNA specific for either $\mathrm{CD} 3 \gamma, \mathrm{CD} 3 \delta$, or a nontarget (NT) control was purchased from Sigma-Aldrich (St. Louis, MO, United States) (Table 1). Lentiviruses were generated by co-transfecting HEK-293T cells (ATCC CRL-1573) with $6.6 \mu \mathrm{g}$ pLKO.1 and the helper plasmids psPAX2 (4.8 $\mu \mathrm{g}$ Addgene \# 12260, containing Gag and Pol) and pMD2.G (1.44 $\mu \mathrm{g}$ Addgene \# 12259, containing VSV-G), kindly provided by Dr. Trono, using Lipofectamine LTX (Life Technologies) according to the manufacturer's instructions. The growth medium was exchanged after $24 \mathrm{~h}$ and the lentiviruscontaining supernatant was harvested 24 and $48 \mathrm{~h}$ later. The cell culture supernatant was used to infect Jurkat E6-1, CH717, or PBL cells in the presence of $8 \mu \mathrm{g} / \mathrm{ml}$ of polybrene (Sigma-Aldrich) or $2 \mu \mathrm{g} / \mathrm{ml}$ of polybrene for the HPB-ALL cell line. After $24 \mathrm{~h}$, the viral particle-containing medium was removed and replaced with fresh medium. After 72h, $2 \mu \mathrm{g} / \mathrm{ml}$ puromycin (Sigma-Aldrich) was added to the Jurkat E6-1, CH717 and HPB-ALL (not for PBL) media for the selection of stable transfectants and tested regularly for $\mathrm{KD}$ status. To generate $\mathrm{CD} 3 \mathrm{KD}$ early thymic progenitors (ETPs) for fetal thymic organ cultures (FTOC), shRNAs were cloned under the U6 promoter into pHRSIN lentiviral vector encoding GFP under the SFFV promoter (pHRSIN-SFFVp-GFP; Garaulet et al., 2013) and HEK-293 cells were co-transfected with the pHRSIN plasmid and the helper plasmids psPAX2 and pMD2.G indicated above. The supernatants were collected after $48 \mathrm{~h}$ of transient transfection and ETPs were transduced on $30 \mu \mathrm{g} / \mathrm{ml}$ recombinant human ( $\mathrm{rh}$ ) fibronectin fragment $\mathrm{CH}$ 296 (RetroNectin) as described (García-Peydró et al., 2003), after overnight culture with rh interleukin 7 (rhIL-7; $100 \mathrm{IU} / \mathrm{ml})$, rh stem cell factor (rhSCF; $100 \mathrm{IU} / \mathrm{ml}$ ), and rh Flt3-ligand (rhFlt3$\mathrm{L} ; 50 \mathrm{IU} / \mathrm{ml}$ ) (National Institute of Biological Standards and Controls, Herts, United Kingdom).

\section{Western Blotting and Immunoprecipitation}

Cells were lysed in NP-40 lysis buffer (1\% NP-40 from SigmaAldrich, 50 mM Tris- $\mathrm{HCl}$ pH 7.4, 100 mM NaCl, 10\% glycerol, 
TABLE 1 | shRNA sequences have been previously published in The RNAi Consortium (TRC) and were purchased from Sigma-Aldrich cloned into pLKO.1 vector.

\begin{tabular}{|c|c|c|}
\hline shRNA & TRC number & Sequence $\left(5^{\prime}-3^{\prime}\right)$ \\
\hline shNT & Nontarget & CAACAAGATGAAGAGCACCAA \\
\hline $\operatorname{shCD} 3 \gamma-1$ & TRCN0000057223 & CGGCTTCCTAACTGAAGATAA \\
\hline $\operatorname{shCD} 3 \gamma-2$ & TRCN0000057224 & CTGGCTATCATTCTTCTTCAA \\
\hline $\operatorname{shCD} 3 \gamma-3$ & TRCN0000057225 & CAGAACTGCATTGAACTAAAT \\
\hline $\operatorname{shCD} 3 \gamma-4$ & TRCN0000057226 & CCACCTTCAAGGAAACCAGTT \\
\hline $\operatorname{shCD} 3 \gamma-5$ & TRCN0000057227 & GTATTACAGAATGTGTCAGAA \\
\hline $\operatorname{shCD} 3 \delta-1$ & TRCN0000057218 & GCATCCATTGAGATGATAATA \\
\hline $\operatorname{shCD} 3 \delta-2$ & TRCN0000057219 & CCGTGCAAGTTCATTATCGAA \\
\hline $\operatorname{shCD} 3 \delta-3$ & TRCN0000057220 & GAGGACAGAGTGTITGTGAAT \\
\hline $\operatorname{shCD} 3 \delta-4$ & TRCN0000057221 & GTTGAGGAATGACCAGGTCTA \\
\hline $\operatorname{shCD} 3 \delta-5$ & TRCN0000057222 & CACTGCTCTCAGACATTACAA \\
\hline
\end{tabular}

$2 \mathrm{mM} \mathrm{MgCl}_{2}, 1 \mathrm{mM}$ PMSF, $1 \mathrm{mM} \mathrm{Na}_{3} \mathrm{VO}_{4}, 25 \mathrm{mM} \mathrm{NaF}$, and $1 \times$ Protease Inhibitor Cocktail Set I-Calbiochem from SigmaAldrich) or Brij96V lysis buffer $(0.3 \%$ Brij96V from SigmaAldrich, 20 mM Tris- $\mathrm{HCl}$ pH 8, $150 \mathrm{mM} \mathrm{NaCl}, 10 \%$ glycerol, $4 \mathrm{mM}$ EDTA, $1 \mathrm{mM}$ PMSF, $2 \mathrm{mM} \mathrm{Na}_{3} \mathrm{VO}_{4}, 10 \mathrm{mM} \mathrm{NaF}$, and $1 \times$ Protease Inhibitor Cocktail Set I-Calbiochem) for WB (Western blot) or IP (immunoprecipitation) experiments, respectively. For IP experiments, $2 \mathrm{mg}$ lysates were incubated for $1 \mathrm{~h}$ at $4^{\circ} \mathrm{C}$ in the presence of $2 \mu \mathrm{g}$ of antibody and protein$\mathrm{G}$ magnetic beads (Dynabeads from Invitrogen, Carlsbad, CA, United States). Precipitates or $40 \mu \mathrm{g}$ of total lysate were loaded in SDS-PAGE gels, transferred into PVDF membranes, and developed with antibodies indicated in each figure. For ER/Golgi transit experiments, half of the immunoprecipitated proteins were digested with recombinant Endoglycosidase $\mathrm{H}$ (EndoH) from Streptomyces plicatus (Sigma-Aldrich), which cleaves within the chitobiose core of high mannose and some hybrid oligosaccharides from $\mathrm{N}$-linked glycoproteins. To this end, samples were incubated with $10 \mathrm{mU}$ EndoH for $3 \mathrm{~h}$ at $37^{\circ} \mathrm{C}$, resuspended in nonreducing loading buffer, and analyzed by WB.

Antibodies against alpha tubulin (Sigma), CD3 $\gamma$ (C20 from Santa Cruz Biotechnology, Dallas, TX, United States, EPR4517 from Abcam, Cambridge, MA, United States, and HMT3.2, kindly donated by Dr. R.T. Kubo, La Jolla Institute for Allergy and Immunology, CA, United States), CD3 $\delta$ (M20 from Santa Cruz, APA 1/2 kindly provided by Dr. B. Alarcón, Centro de Biología Molecular Severo Ochoa, Madrid, Spain, and EP4426 from Abcam), CD3 $\varepsilon$ (EPR5361(2) from Abcam), or $\zeta \zeta / C D 2472$ (448, kindly provided by B. Alarcon, Centro de Biología Molecular Severo Ochoa, Madrid, Spain) were used. Blots were visualized using Odyssey Infrared Imaging System (LI-COR Biosciences, Lincoln, NE, United States). Images of IRDye680 and IRDye800 fluorescence were obtained using the 700- and 800-nm channels. The quantification of signal intensity was carried out with Image Studio software.

\section{Flow Cytometry}

Multiparametric flow cytometry was performed with standard procedures. For extracellular flow cytometry, monoclonal antibodies against CD3 (clone HIT3A APC and SK7 PE from Becton Dickinson, Franklin Lakes, NJ, United States), CD3ع (UCHT-1 PE-Cy5 from Beckman Coulter, Brea, CA, United States; Hit3A-APC from BioLegend, San Diego, CA, United States), TCR $\alpha \beta$ (BMA031 APC from Miltenyi, Auburn, CA, United States), TCR $\gamma \delta$ (clone 5A6.E9 Pe-Cy5 from Life Technologies), V 33 (CH92 FITC from Beckman Coulter), V $\beta 8$ (56C5 PE from Beckman Coulter), C $\beta 1$ (JOVI-1 sc-53196 from Santa Cruz Biotechnology), CD2 (S5.2 APC from BD), and HLA-class I (W6/32 PE-Cy7 from eBioscience, San Diego, CA, United States) were used. For intracellular staining, cells were fixed with $2 \% \mathrm{PFA}$ for $30 \mathrm{~min}$ at $4^{\circ} \mathrm{C}$, permeabilized with $0.2 \%$ saponin for $15 \mathrm{~min}$ at $20-22^{\circ} \mathrm{C}$ and stained with anti-CD3 $\gamma$ or anti-CD3 $\delta$ (clones EPR4517 or EP4426 from Abcam) followed by a secondary fluorescent antibody (anti-rabbit PE from Beckman Coulter). Cells were stained with one single antibody per tube, labeled cells were acquired on FACSCalibur flow cytometer (Becton Dickinson) and the data analyzed with FlowJo software (TreeStar, Ashland, OR, United States).

\section{Fluorescence Microscopy}

Cells were seeded in circular cover glasses, preincubated with $0.005 \%$ poly-L-lysine (Sigma-Aldrich) for $30 \mathrm{~min}$ at $37^{\circ} \mathrm{C}$, and rinsed several times. Cells were then fixed with $4 \%$ p-formaldehyde in PBS for $5 \mathrm{~min}$ and, after rinsing, permeabilized with $0.1 \%$ Triton X-100 (Sigma-Aldrich) in PBS (both steps at room temperature, $20-22^{\circ} \mathrm{C}$ ). Nonspecific binding sites were blocked by incubation for $1 \mathrm{~h}$ in PBS containing $10 \mu \mathrm{g} / \mathrm{ml}$ human IgG and a commercial blocking reagent (Roche, Basel, Switzerland). Incubation with primary TCR $\beta$ antibody (JOVI-1) or secondary anti-mouse antibody AF594 (Invitrogen) was done for $1 \mathrm{~h}$ or $30 \mathrm{~min}$ at $20-22^{\circ} \mathrm{C}$, respectively. Appropriate isotype-matched IgG or normal serum was used in parallel as the control. Stained cells were mounted in Vectashield ${ }^{\circledR}$-DAPI solution (Vector Laboratories, Burlingame, CA, United States) and visualized under a Leica SP-2 AOBS confocal microscope (HCX PL APO × 631.4 oil-immersion objective; 1.400,000, numerical aperture) and TCSNTV software (Leica Microsystems, Wetzlar, Germany). Images were software processed with ImageJ.

\section{Statistical Analysis}

Statistical analysis, including regression comparisons, was performed using GraphPad software. All quantitative data are expressed as the mean \pm standard deviation (SD). Statistical differences between groups were compared using one-sample Student's $t$-test. $P<0.05\left(^{*}\right)$ was considered to indicate a statistically significant result. ${ }^{* * *} P<0.01$.

\section{Isolation of Human Thymocytes and Hybrid Human-Mouse Fetal Thymic Organ Cultures}

Experiments were performed in accordance with the procedures approved by the Spanish Research Council Bioethics Committee. Normal human postnatal thymocytes were isolated from thymus fragments removed during corrective cardiac surgery of patients aged 1 month to 4 years, after informed consent in accordance 
with the Declaration of Helsinki. Thymocyte suspensions were subjected to density centrifugation using Ficoll-Hypaque (Lymphoprep, Axis-Shield PoC AS), and Early T-cell progenitors (ETPs) were then isolated by immunomagnetic cell sorting using the Dynal CD34 Progenitor Cell Selection System (Life Technologies). Fetal thymic organ culture (FTOC) assays were performed as described (García-Peydró et al., 2003). Briefly, thymic lobes from 14.5-day-old Swiss mouse embryos were seeded (one lobe/well) in Terasaki plates (Nunc, Roskilde, Denmark), treated with deoxyguanosine (d-Guo, Sigma), and co-cultured with shNT- or shCD3 8 -transduced human ETPs (1$2 \times 10^{4}$ cells/lobe). After $48 \mathrm{~h}$ of culture in hanging drops (day 0 of FTOC), lobes were cultured on gelfoam/filters for up to 5 weeks. T-cell generation was analyzed by flow cytometry on electronically gated $\mathrm{GFP}^{+}$cells recovered from different lobes pooled at indicated time points.

\section{RESULTS}

\section{Generation and Characterization of CD3G or CD3D KD T-Cell Lines}

To investigate the importance of $\operatorname{CD} 3 \gamma$ and $\operatorname{CD} 3 \delta$ in TCR assembly and expression, we generated $C D 3 G$ or $C D 3 D \mathrm{KD}$ T-cell lines using five different shRNA sequences for inhibition of CD3 expression (Table 1). The Jurkat E6-1 T-cell line was used to screen the different shRNAs. To this end, cells were transduced with lentiviral vectors carrying shRNA specific for CD3 $\gamma(\operatorname{shCD} 3 \gamma), \mathrm{CD} 3 \delta(\operatorname{shCD} 3 \delta)$, or a nontarget shRNA (shNT). After at least 7 days in culture, cells were lysed in the presence of the selective agent puromycin and analyzed by WB for the expression of the targeted CD3 chain. Band intensity analysis showed that different sequences led to different degrees of KD (Figure 1). For further analysis, we selected lines shCD $3 \gamma-1$ and $\operatorname{shCD} 3 \delta-3$, for the most efficient inhibition relative to shNT.

To fully characterize the impact of the KD on the TCR, we analyzed in stable KD T-cell lines the level of expression of all CD3 chains by WB. The results showed a marked selective impairment in the expression of CD $3 \gamma$ or CD $3 \delta$ proteins in Jurkat cells treated with shCD $3 \gamma$ or shCD $3 \delta$, respectively, as compared with shNT (Figures 2A,B). The analysis of CD3 $\varepsilon$ expression in $\mathrm{KD}$ lines showed a slight but significant $30 \%$ reduction of $\mathrm{CD} 3 \varepsilon$ in the $C D 3 G$, but not in the $C D 3 D, K D$ cell line (Figure 2C).

\section{TCR Assembly in CD3G or CD3D KD T-Cell Lines}

To evaluate the impact of the KD of $\operatorname{CD} 3 \gamma$ or $\operatorname{CD} 3 \delta$ chains on TCR assembly, we analyzed TCR stoichiometry by coimmunoprecipitation (co-IP) with anti-TCR $\beta$ and WB with CD3 chain-specific antibodies. Compared with shNT cells, when $C D 3 G$ was KD only $51 \%$ of CD $3 \varepsilon$ and $18 \%$ of CD $3 \delta$ co-IPed with TCR $\beta$ (Figures 3A,B, top lanes). However, when CD3D was KD, similar amounts of CD3 $\varepsilon(101 \%)$ and higher amounts of CD3 $\gamma$ (152\%) were incorporated (Figures 3A,C top lanes). Thus, higher amounts of $\gamma \varepsilon$ dimers were incorporated to $(\alpha) \beta$ after $C D 3 D \mathrm{KD}$ than were $\delta \varepsilon$ dimers after $C D 3 G$ KD (summarized in Figure 3D).

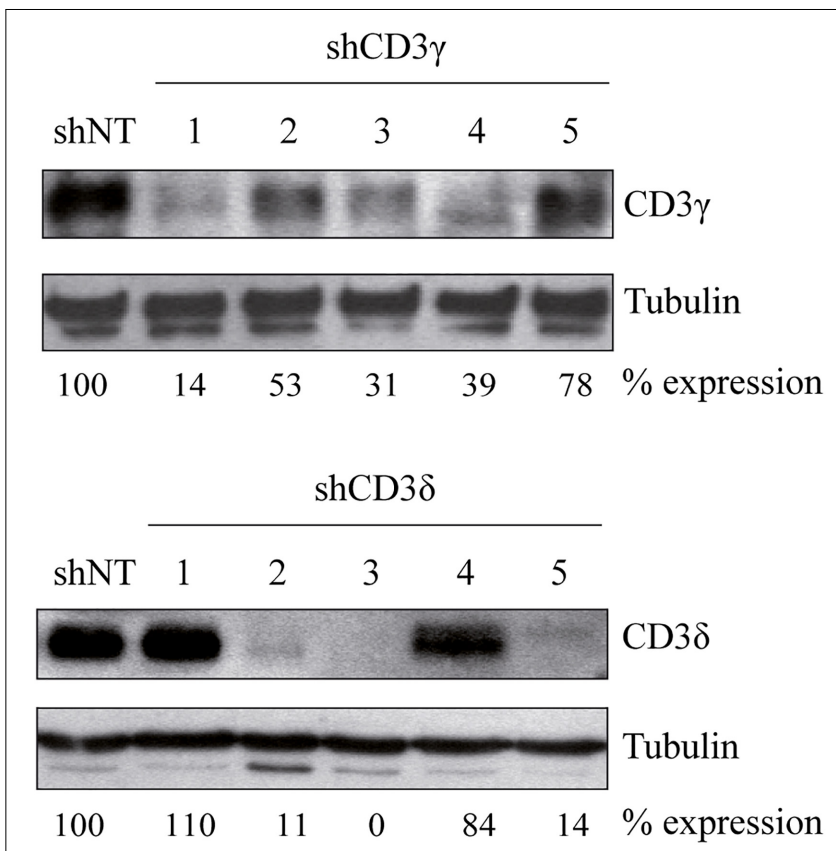

FIGURE 1 | Generation of CD3G or CD3D KD T-cell lines. Jurkat E6-1 cells were transduced with five different shRNA for CD3 $\gamma$ or CD3 $\delta$ and one non-target shRNA (shNT). Cells were lysed in NP-40 buffer after 7 days in culture and $40 \mu \mathrm{g}$ of total protein was loaded. Membranes were incubated with antibodies against alpha tubulin (Sigma), CD3 $\gamma$ (Santa Cruz Biotechnology), or CD3 $\delta$ (Santa Cruz Biotechnology). Numbers below each panel indicate the percentage of each chain expression relative to shNT normalized to the loading control (tubulin).

We conclude that TCR ensembles are more stable when CD3 $\delta$, rather than $\mathrm{CD} 3 \gamma$, is scarce.

\section{TCR ER/Golgi Transit in CD3G or CD3D KD T-Cell Lines}

To determine the subcellular compartment location of the remaining CD3 chains after the KD, we studied TCR ER/Golgi transit by analyzing $\mathrm{CD} 3$ chain sensitivity to EndoH after IP and WB with CD3 chain-specific antibodies, because the Golgi, but not the ER, protects CD3 oligosaccharide side chains from EndoH digestion. Both forms of $\mathrm{CD} 3 \gamma$ and $\mathrm{CD} 3 \delta$, post-ER (EndoH resistant) and ER resident (EndoH sensitive), can be observed in shNT cells (Figure 4). In contrast, CD3D KD essentially blocked $\mathrm{CD} 3 \gamma$ in the ER (only 3\% exited the ER, Figure 4A). Similarly, in CD3G KD cells, only $10 \%$ of CD3 $\delta$ was able to reach the Golgi (Figure 4B). Our results indicate that both $\mathrm{CD} 3 \gamma$ and $\mathrm{CD} 3 \delta$ chains in both $\mathrm{KD}$ cell lines were EndoH sensitive and, therefore, ER resident, suggesting that TCR complexes in the absence of CD $3 \gamma$ or CD $3 \delta$ are retained in the ER.

\section{$\zeta \zeta / C D 247_{2}$ Incorporation to the TCR in CD3G or CD3D KD T-Cell Lines}

To study the last step in TCR assembly, namely, $\zeta \zeta / C D 247_{2}$ incorporation, its association to TCR $\beta$ and $\operatorname{CD} 3 \varepsilon$ was analyzed by IP and WB. The results showed that the clear co-IP of TCR $\beta$ 

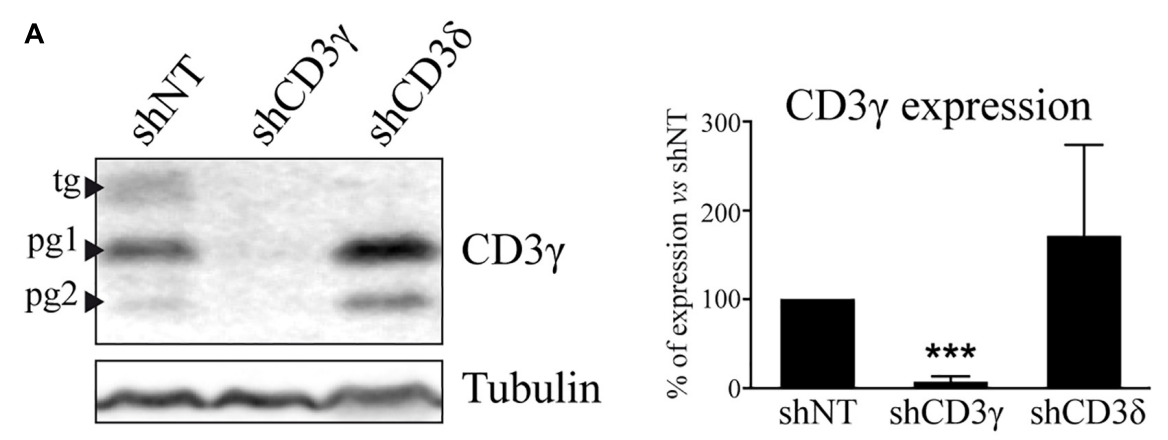

B

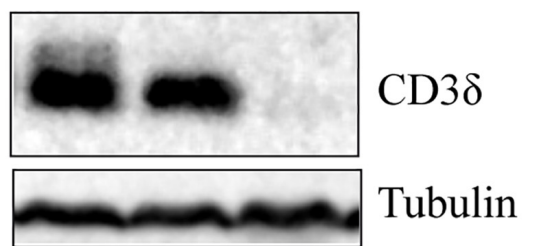

C

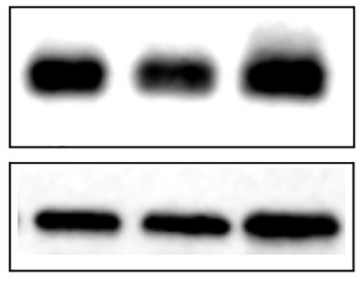

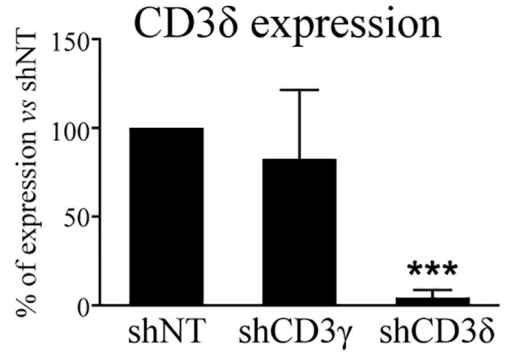

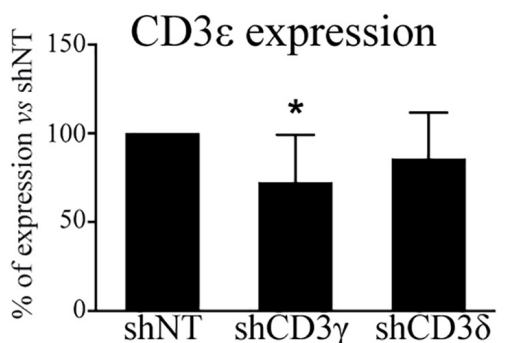

FIGURE 2 | CD3 protein expression in CD3G or CD3D KD T-cell lines. Jurkat E6-1 cells were transfected with shCD3 $\gamma$, shCD38, and shNT. Cells were lysed in NP-40 buffer after several days in culture with puromycin and $40 \mu \mathrm{g}$ of total protein was loaded. Membranes were incubated with antibodies against tubulin (Sigma) and (A) CD3 $\gamma$ (Abcam), (B) CD3 $\delta$ (Santa Cruz Biotechnology), or (C) CD3 $\varepsilon$ (Abcam). Band analysis and numbers as in Figure 1. Left: a representative experiment. Right: quantification of at least 10 different experiments; data are mean values + SD. Tg, totally glycosylated; pg, partially glycosylated. Note: all glycosylated forms of CD3 $\gamma$ were quantified together.

and CD247 (Figure 5A) and of CD247 and CD3 $\varepsilon$ (Figure 5B) observed in shNT cells was absent in both $C D 3 G$ and $C D 3 D$ KD T-cell lines. We conclude that TCR complexes in both CD3 KD cells do not incorporate $\zeta \zeta / C D 247_{2}$ homodimers.

\section{Surface Expression of the TCR Complex in CD3G or CD3D KD T-Cell Lines}

To assess the impact of $C D 3 G$ or $C D 3 D \mathrm{KD}$ on surface TCR expression, flow cytometry was performed using a broad panel of $\mathrm{mAb}$ against the TCR or CD3 chains in the Jurkat E6-1 KD and in a HVS CD $3 \gamma^{-/-}$cell line. The results indicated that the KD cell lines with reduced intracellular amounts of either CD $3 \gamma$ or CD3 $\delta$, expressed decreased surface TCR levels detected with several antibodies, with between 5 and $10 \%$ of surface TCR relative to controls in both cell lines (Figure 6). These observations demonstrated a critical requirement of $\operatorname{CD} 3 \gamma$ and $\operatorname{CD} 3 \delta$ for surface TCR expression in the Jurkat E6-1 T-cell line. The effects of CD3 KD on surface TCR levels were also analyzed in primary $\mathrm{T}$ cells and T-cell lines other than Jurkat E6-1 (Jurkat CH7C17 and HPB-ALL), and the results were comparable to Jurkat E6-1 $\mathrm{T}$ cells (Figure 7), further supporting that $\mathrm{CD} 3 \gamma$ and $\mathrm{CD} 3 \delta$ are required for normal surface TCR expression in mature T cells. These results were confirmed by confocal microscopy for TCR $\beta$, which was detectable both intracellularly and extracellularly in Jurkat E6-1 shNT cells, but only intracellularly in CD3G and CD3D KD cell lines (Figure 8). The shNT unpermeabilized cells stained for TCR $\beta$ showed clear evidence of patching, likely as a result of crosslinking with the secondary antibody (see Figure $\mathbf{8}$ legend). In contrast, TCR $\beta$ was absent on the cell surface of both CD3 KD cells. Surprisingly, surface TCR was less affected in the HVS CD $3 \gamma^{-/-}$cell line than in KD lines (Figure 6). We conclude that TCR complexes do not reach the surface in any of the CD3 KD cells. Therefore, the stark differences in disease presentation in patients lacking $\mathrm{CD} 3 \gamma$ (mild) or $\mathrm{CD} 3 \delta$ (severe) cannot be ascribed to differences in the role of each chain in mature T cells. 
A

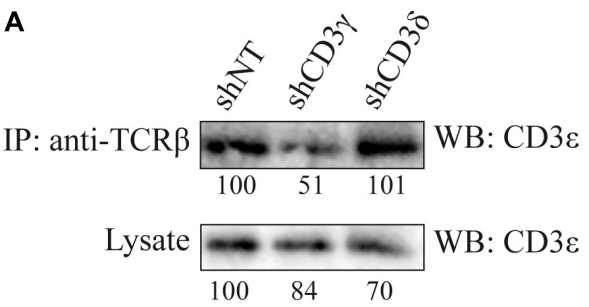

Lysate

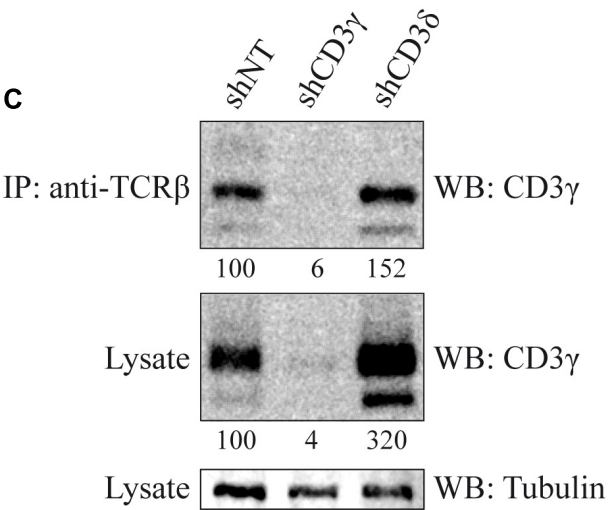

B

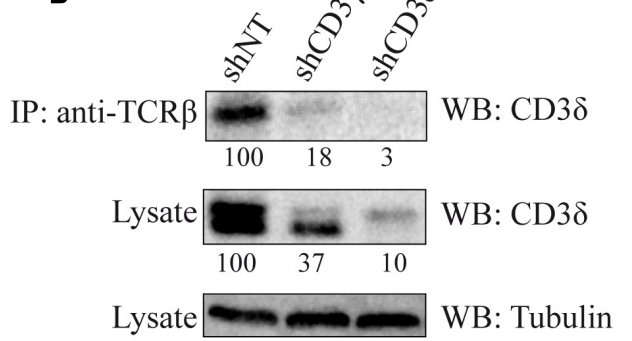

D co-IP with anti-TCR $\beta$

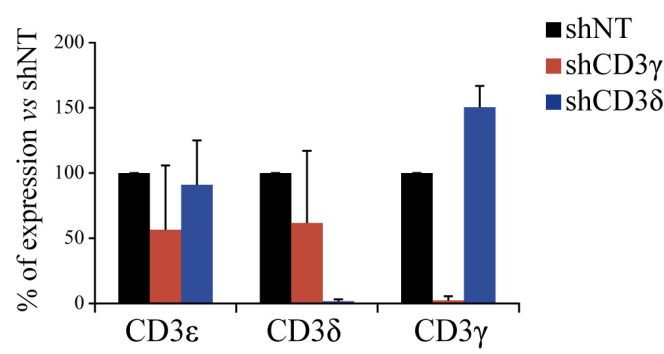

FIGURE 3 | Co-IP of TCR $\beta$ and CD3 chains in CD3G or CD3D KD T-cell lines. Cells were lysed in Brij96V buffer, and 3 mg of total protein was immunoprecipitated (IP) with the anti-TCR $\beta$ mAb JOVI-1; the immunoprecipitates and whole lysates were resolved in 15\% SDS-PAGE gels, transferred into PVDF membranes, and immunoblotted with antibodies against alpha tubulin and CD3 $\varepsilon$ in (A), CD38 in (B), or CD3 $\gamma$ in (C). Analysis and numbers as in Figure 1. (D) Quantification of three different experiments; data are mean values + SD.

A

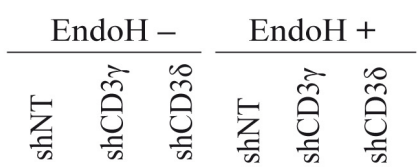

IP: anti-CD3 $\gamma$

WB: CD3 $\gamma$

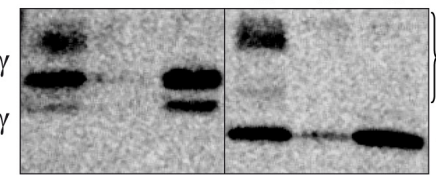

$\rightarrow$ EndoH Resistant

$\rightarrow$ EndoH Sensitive

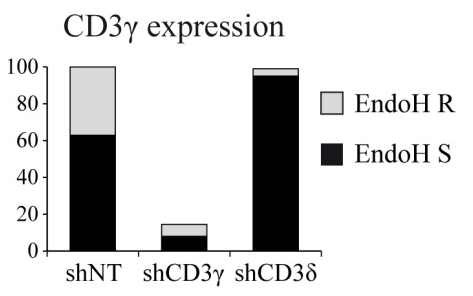

B

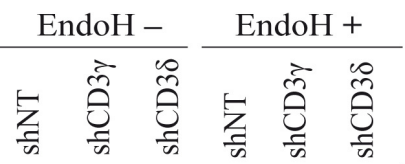

IP: anti-CD3 $\delta$

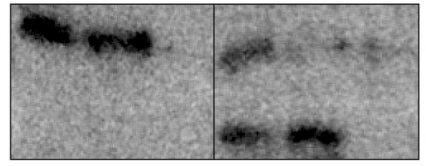

$\rightarrow$ Endo-H Resistant

$\rightarrow$ Endo-H Sensitive
CD3 $\delta$ expression

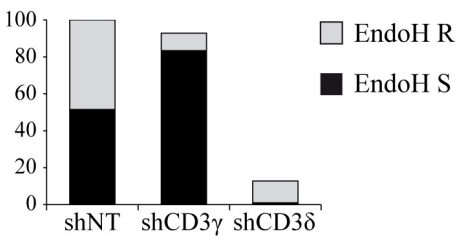

FIGURE 4 | EndoH sensitivity of CD3 chains in CD3G or CD3D KD T-cell lines. (A) Cells were lysed in Brij96V buffer and immunoprecipitated with anti-CD3 $\gamma$ (HMT3.2). Half of the immunoprecipitated proteins were treated with EndoH. Samples were analyzed in 15\% SDS-PAGE gels and immunoblotted with anti-CD3 $\gamma$ antibody (Abcam). The graph represents the quantification of EndoH-resistant (R, Golgi-associated) and EndoH-sensitive (S, ER-associated) species. (B) Cells were immunoprecipitated with anti-CD3 (APA1/2) antibody and immunoblotted with anti-CD3 (Abcam). Sample preparation, treatment with EndoH, and analysis as in (A). 


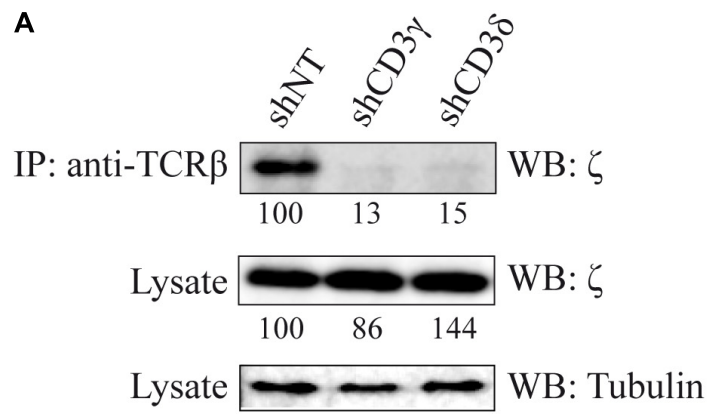

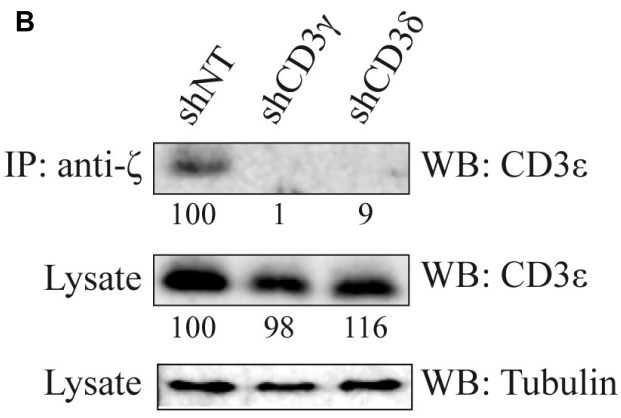

FIGURE 5 | Co-IP of $\zeta \zeta / C D 2472$ and other TCR chains in CD3G or CD3D KD T-cell lines. (A) Cells were lysed in Brij96V buffer, 3 mg of total protein was immunoprecipitated with anti-TCR $\beta \mathrm{mAb}$ (JOVI-1), and the immunoprecipitates and whole lysates were resolved in 15\% SDS-PAGE gels and immunoblotted with antibodies against $\zeta \zeta / C D 2472$ (448) or alpha tubulin (Sigma). (B) Immunoprecipitation was done with anti- $\zeta$ and immunoblot with mAb against CD3 $\varepsilon$ (Abcam) or $\alpha$-tubulin (Sigma). Band analysis and numbers as in Figure 1. The tubulin loading control is shared in (A,B) for illustrative purposes.

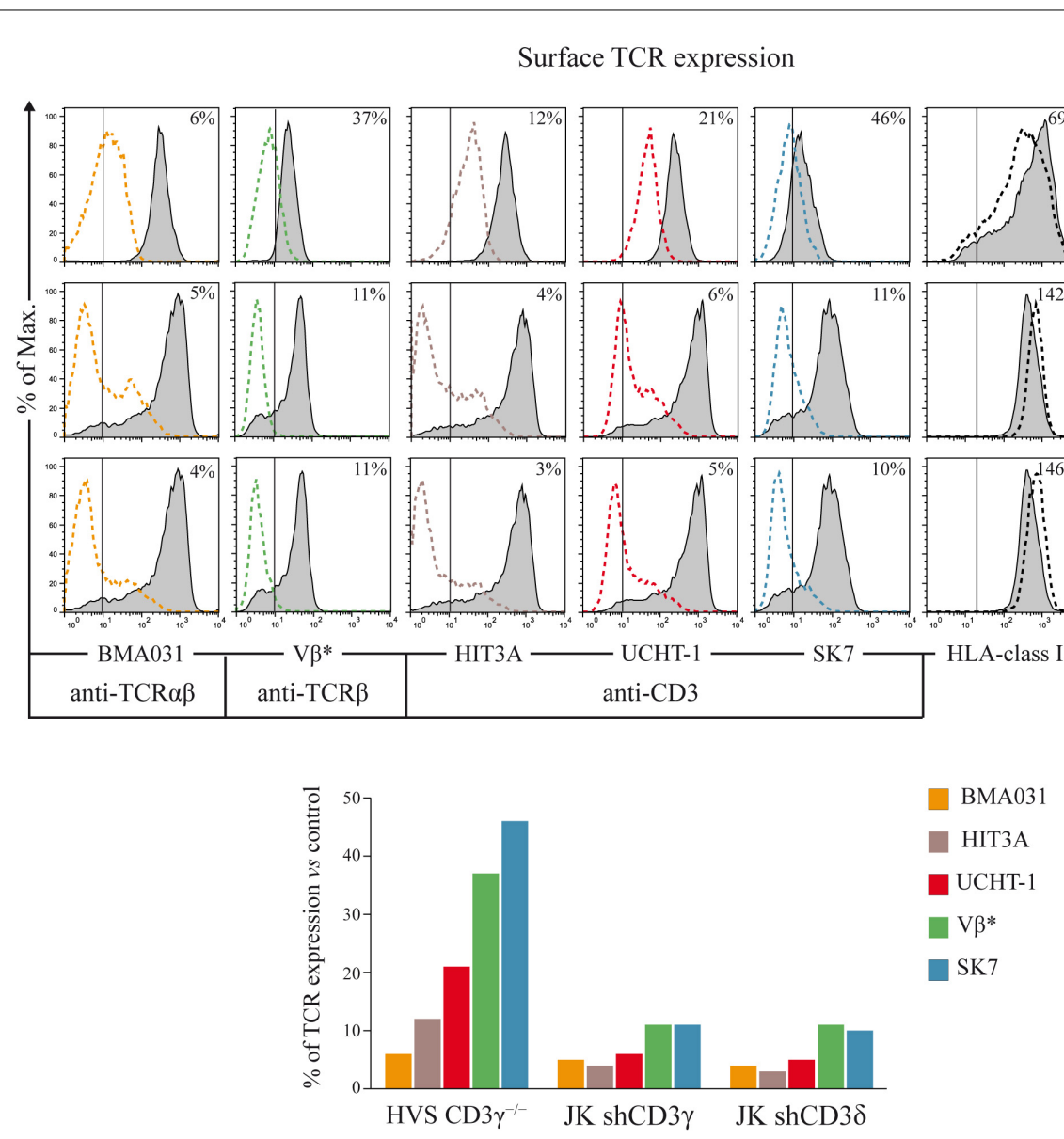

FIGURE 6 | Surface TCR expression in CD3G or CD3D KD T-cell lines. Top: cells were stained with labeled TCR- or CD3-specific mAb (BMA031, HIT3A, UCHT-1, $V \beta^{*}$, and SK7) or with TCR-independent control mAb (anti-HLA-class I and anti-CD2). Expression was analyzed in HVS-CD3 $\gamma^{-/-}$, Jurkat shCD3 $\gamma$, and Jurkat shCD3 $\delta$ cells (dashed lines) compared with their relevant controls (HVS-CD $3 \gamma^{+/+}$or Jurkat shNT, gray histograms, the latter shown twice as it is the control cell line for both CD3G and CD3D KD cell lines). The vertical line in each panel indicates the upper limit of background fluorescence using isotype-matched irrelevant mAb. The numbers in each histogram indicate geoMFI ratios $(\times 100)$ relative to controls. Bottom: bar representation of TCR/CD3 geoMFI ratios relative to controls. ${ }^{*} / \beta 3$ or V $\beta 8$-specific mAb was used to stain the TCR $\beta$ chain in HVS or Jurkat cells, respectively, because they have different TCRB rearrangements. 


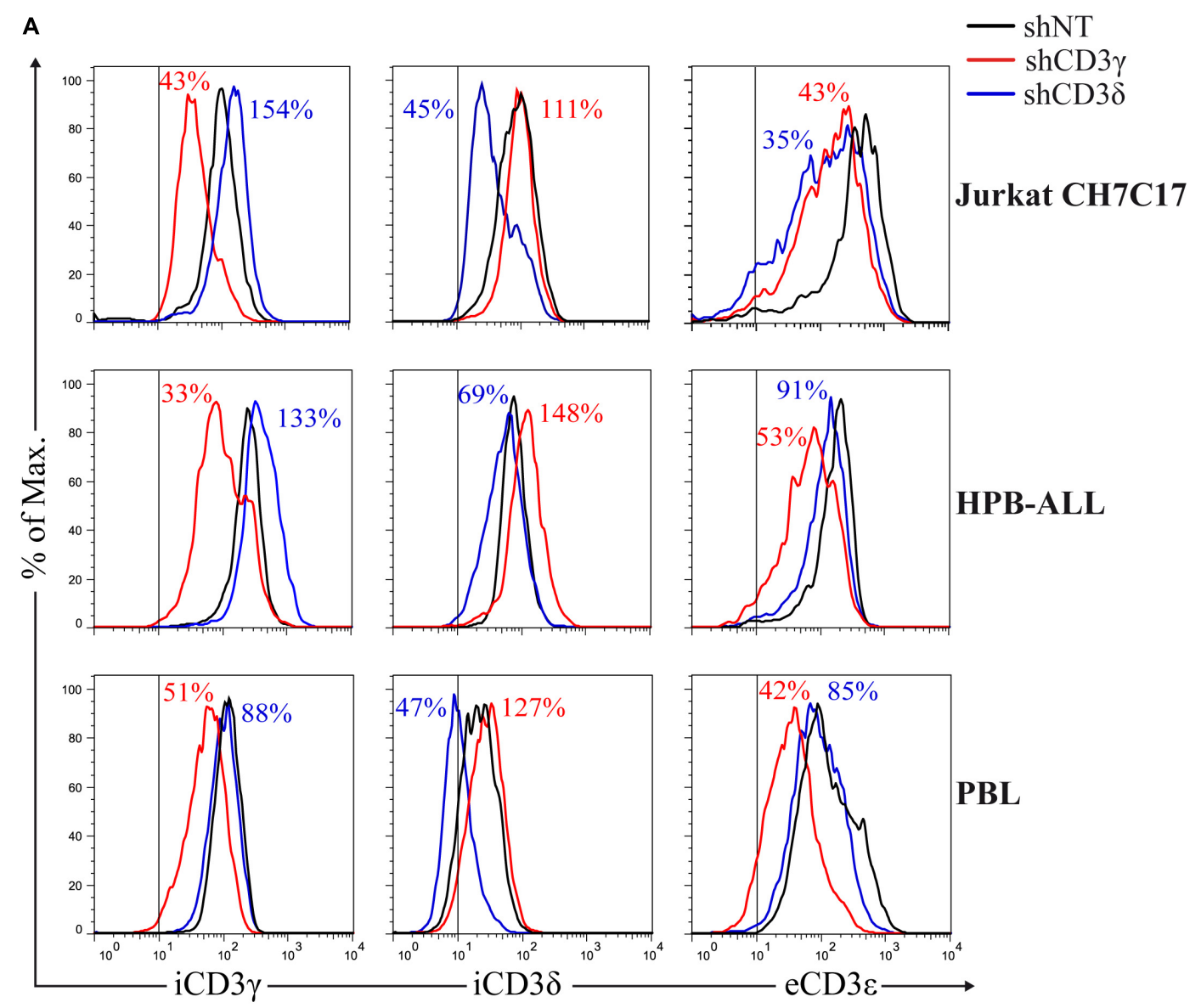

B

- Jurkat E6-1

- Jurkat $\mathrm{CH} 7 \mathrm{C} 17$

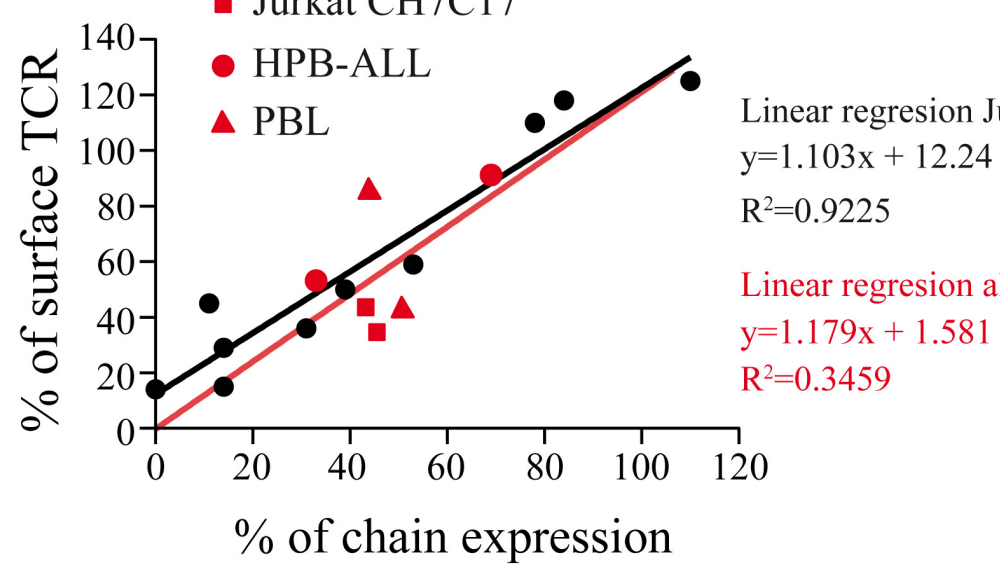

FIGURE 7 |CD3 KD and surface TCR expression correlate in T cells other than Jurkat E6-1. (A) Comparative intracellular (i) CD3 $\gamma$ or CD3 expression and

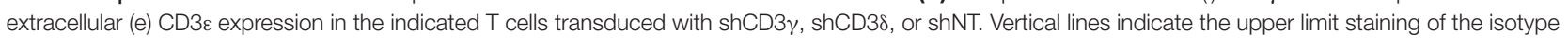
control. Numbers indicate \% expression vs. control (shNT). (B) Correlation between CD3 $\gamma$ or CD3 $\delta$ chain expression and surface TCR, expressed as \% of expression vs. shNT. Data in (A) (red symbols) in comparison with Jurkat E6-1 data (black dots) and comparative linear regression. The slopes are not significantly different $(P=0.9046)$. 


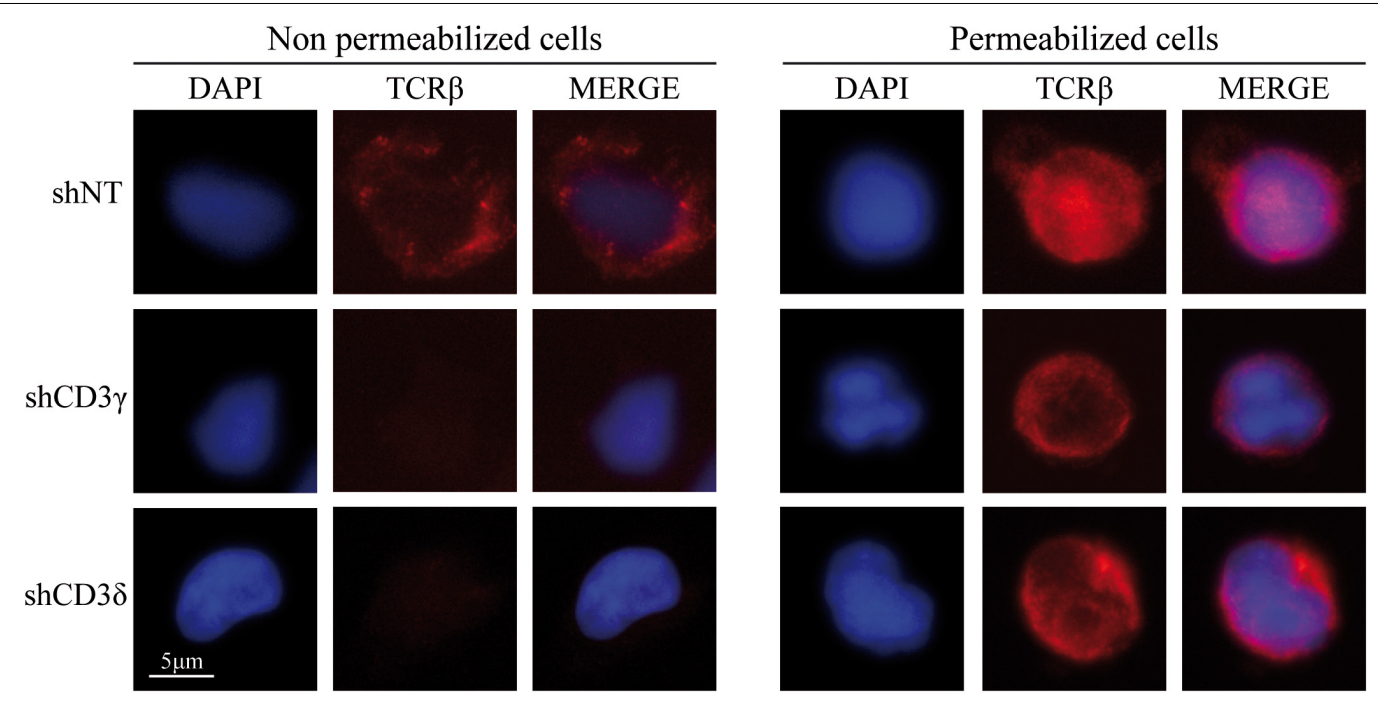

FIGURE 8 | Extracellular (left) and intracellular (right) TCR $\beta$ expression in CD3G or CD3D KD T-cell lines. Jurkat E6-1 shNT, shCD3 $\gamma$, and shCD3 $\delta$ cells were left untreated (unpermeabilized) or were treated with Triton X-100 (permeabilized) and incubated with anti-TCR 3 antibody (JOVI-1) and anti-mouse AF594 secondary antibody before analysis by fluorescence microscopy. Nuclei were stained with DAPI.

Rather, there must be critical signaling or expression differences whereby $\operatorname{CD} 3 \gamma$, but not $\mathrm{CD} 3 \delta$, can be replaced early in T-cell development. Indeed, as explained below, $\mathrm{CD} 3 \delta$, but not $\mathrm{CD} 3 \gamma$ deficiency, showed a complete block in the differentiation of $\mathrm{T}$ cells in the patient's thymus (Dadi et al., 2003).

\section{Surface Expression of the TCR Complex in CD3D KD Early T-Cell Progenitors}

Our results in human mature $\mathrm{T}$ cells showed that both $\mathrm{CD} 3 \gamma$ and $\mathrm{CD} 3 \delta$ are required for surface TCR expression. Our coIP experiments showed that nascent TCRs begin to form but are unable to incorporate $\zeta \zeta / \mathrm{CD} 247$ dimers and are retained in the ER. These results do not recapitulate the corresponding congenital CD3 deficiencies in terms of surface TCR expression ( $>30 \%$ vs. normal controls). We thus hypothesized that intrathymic plasticity of emerging polyclonal lymphocytes in patients might allow some TCR expression in a proportion of $\mathrm{T}$ cells, which could then signal for survival in CD3 $\gamma$, but not in CD3 $\delta$ deficiency. To directly address this hypothesis, we transduced early $\mathrm{T}$-cell progenitors from human thymus with shCD $3 \delta-3$ or shNT before FTOC. We chose this KD strategy in order to assess both T-cell development and TCR expression in a single experiment, since shCD3 $\gamma$ would be expected to be milder in both aspects. The results indicated that $C D 3 D \mathrm{KD}$ strongly impaired human T-cell development in FTOC (Figure 9, top and middle), as observed also in human congenital CD3 $\delta$ deficiencies. However, the few T cells (pre-T, $\alpha \beta$, or $\gamma \delta$ ) that did emerge from the organ cultures expressed comparable, albeit lower (around $80 \%$ vs. shNT or control KD), TCR ensemble levels. These results support the hypothesis of intrathymic TCR expression plasticity of emerging polyclonal $\mathrm{T}$ lymphocytes, which can then signal for survival in $\operatorname{CD} 3 \gamma$, but not in $\operatorname{CD} 3 \delta$ deficiency, and explain the immunological and clinical disparities of these ID cases.

\section{DISCUSSION}

In contrast with all other reported CD3 ID due to complete protein deficiencies, most $\mathrm{CD} 3 \gamma$-deficient individuals can live well into their 30 s without hematopoietic stem cell replacement (Marin et al., 2015). In these individuals, late diagnosis, mild $\mathrm{T}$ lymphopenia, low but significant TCR expression, and autoimmune features are common. These limited immunological consequences are at odds with what is observed when the highly homologous $\mathrm{CD} 3 \delta$ chain is absent: early diagnosis, severe $\mathrm{T}$ lymphopenia, no TCR expression, and urgent transplantation. This suggests that there must be strong biochemical or functional differences between them. Conversely, mouse KO models show that $\operatorname{CD} 3 \gamma$, but not $\operatorname{CD} 3 \delta$, is critical for early thymic development, and therefore, do not recapitulate human findings. We reasoned that by $\mathrm{KD}$ of each chain in human mature $\mathrm{T}$ cells we could find relevant differences. However, we found that both $C D 3 G$ and $C D 3 D \mathrm{KD}$ T-cell lines were unable to incorporate $\zeta \zeta / C D 247_{2}$ to their TCR, and thus, showed a comparable decrease in surface TCR expression. We also observed in the analysis of ER/Golgi transit that $C D 3 G$ and $C D 3 D$ KD completely blocked TCR ensembles since CD $3 \delta$ and CD $3 \gamma$, respectively, did not reach the Golgi. Therefore, the immunological and clinical disparities of ID cases may be associated with biochemical or signaling differences between CD $3 \gamma$ - and CD $3 \delta$-deficient TCR ensembles in immature rather than mature $\mathrm{T}$ lymphocytes, for instance, at the pre-TCR level. To support this hypothesis, analysis of gene expression in thymocytes isolated from one patient diagnosed with SCID caused by CD3 $\delta$ deficiency showed that a small number of gene products known to regulate $\mathrm{T}$-cell development were substantially altered in the patient, as compared with the control. Of particular interest, patient's thymocytes contained twice as much precursor T-cell receptor a (pTa) gene transcript as did control thymocytes. Since the pTa gene is expressed 


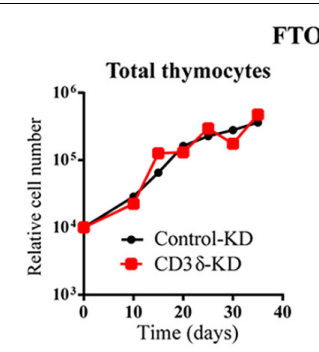

FTOC \#1
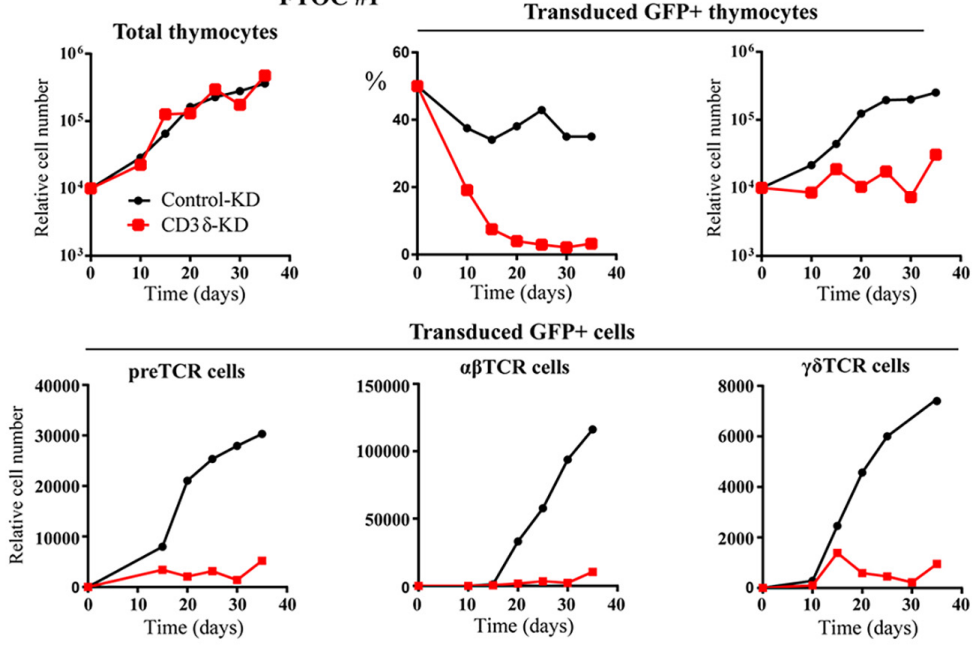

Transduced GFP+ cells
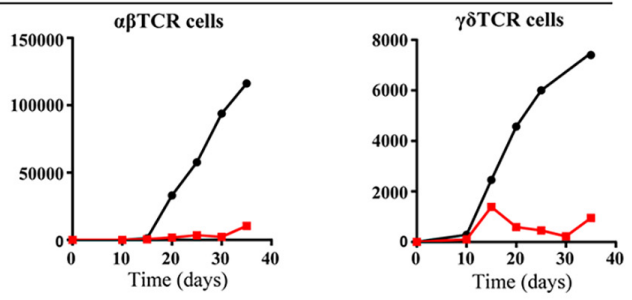

FTOC \#2
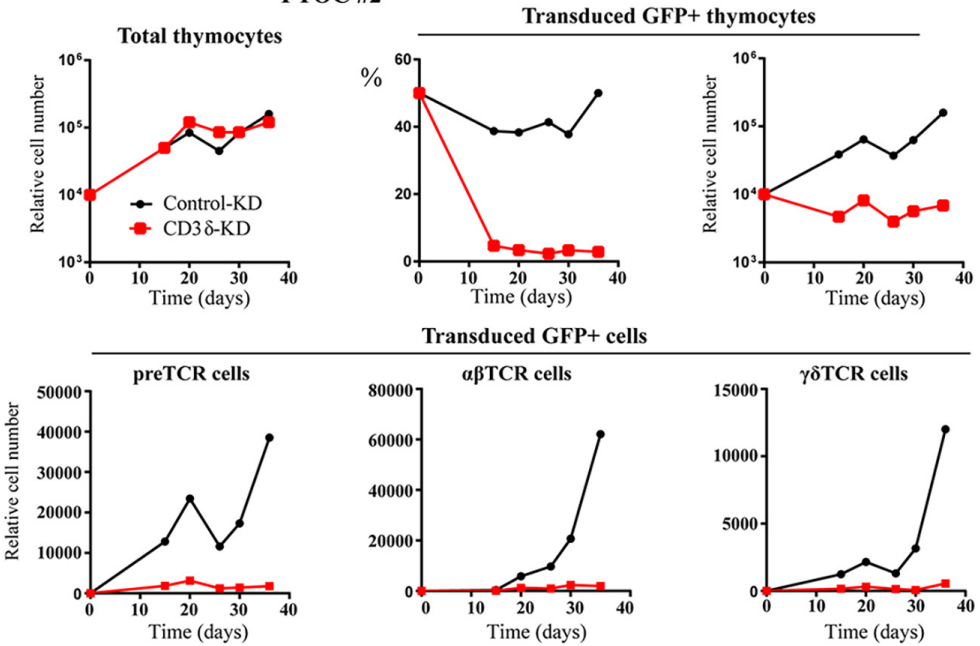

Transduced GFP+ cells
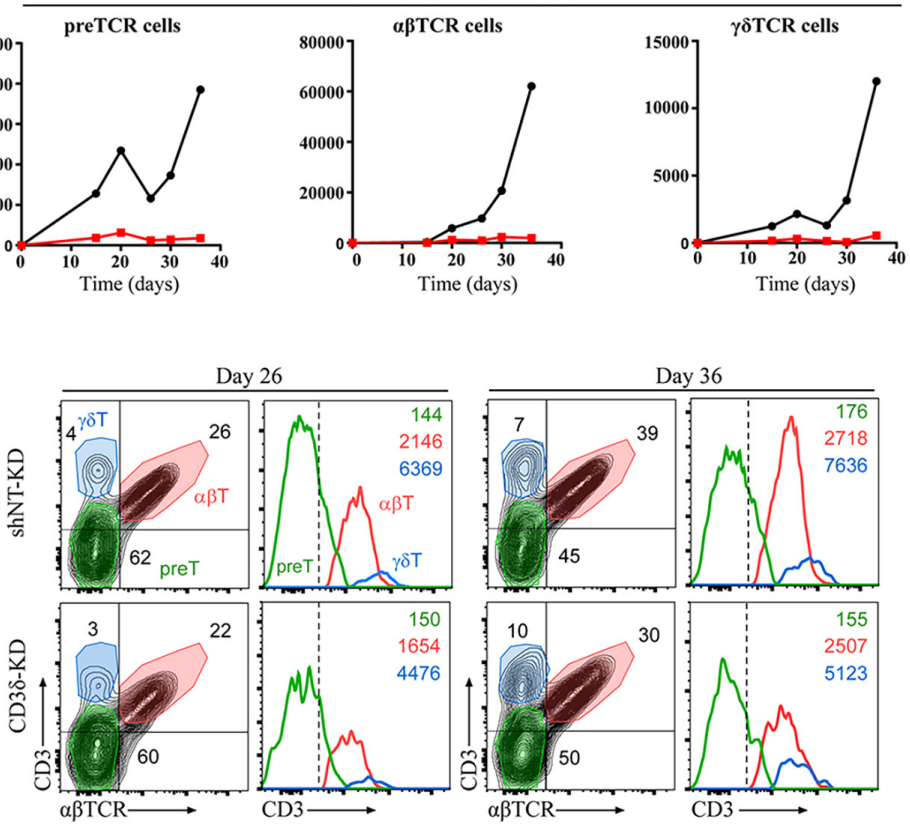

FIGURE 9 | Fetal thymus organ cultures (FTOC) of shCD3 $8-3$ vs. shNT-transduced human T-cell progenitors. Top and middle: mouse embryo thymic lobes were seeded with shCD38-3 or shNT-transduced CD34 ${ }^{\text {hi }}$ early T-cell progenitors from human thymus and cultured for up to 5 weeks. T-cell generation was analyzed on electronically gated $\mathrm{GFP}^{+}$transduced cells recovered from different lobes pooled at the indicated time points in two independent experiments. Bottom: representative (FTOC 2) flow cytometry analysis of CD3 vs. $\alpha \beta T C R$ expression on electronically gated GFP+ cell progenies at the indicated days. Numbers in

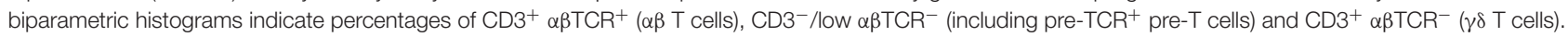
Percentages of $\gamma \delta$ T cells were independently confirmed by CD3 vs. $\gamma \delta$ TCR expression analysis. Numbers in monoparametric histograms indicate MFI values for each indicated subset. 
exclusively by immature thymocytes, these results indicate a block early in the differentiation of T cells in the patient's thymus. Such a block could cause immature $\mathrm{CD} 4^{-} \mathrm{CD} 8^{-}$double-negative thymocytes to accumulate (Dadi et al., 2003).

The analysis of $\mathrm{CD} 3 \varepsilon$ expression in $\mathrm{KD}$ lines showed a slight but significant reduction of $C D 3 \varepsilon$ in the $C D 3 G$, but not in the $C D 3 D, \mathrm{KD}$ cell line (Figure 2) and less stable $(\alpha) \beta \delta \varepsilon$ than $(\alpha) \beta \gamma \varepsilon$ TCR ensembles (Figure 3). A potential explanation for both events may be the faster degradation of $(\alpha) \beta \delta \varepsilon$ TCR compared with $(\alpha) \beta \gamma \varepsilon$ TCR. However, such differences did not have an impact on later assembly events or surface TCR expression, which were similar in both KD cell lines.

TCR ensembles barely reached the T-cell surface in any of the CD3 KD Jurkat cells ( $<11 \%$ of normal controls). A mutagenized Jurkat T-cell line lacking CD3 $\gamma$ called JGN (for Jurkat Gamma Negative; Geisler, 1992) also lacked surface TCR expression, supporting our findings. However, surface TCR was much less affected in the CD3 $\gamma$ ID-derived HVS T-cell line than in our $\mathrm{KD}$ lines or in JGN ( $>30 \%$ vs. normal controls), and this is the case too for the scarce peripheral mature $\mathrm{T}$ cells in partial CD3 $\delta$ ID (Gil et al., 2011; Garcillán et al., 2014). Why does natural $\mathrm{CD} 3 \gamma$ deficiency differ from Jurkat CD3 $\gamma \mathrm{KD}$ ? Our hypothesis is that mature $\mathrm{T}$ cells in $\mathrm{CD} 3 \gamma$ deficiency are the result of selection from a very diverse and polyclonal repertoire of immature $\mathrm{T}$ cells with differently rearranged variable TCR chains, so that those expressing sufficient surface TCR can be selected to reach the peripheral blood compartment, albeit selftolerance may be affected (Rowe et al., 2018). By contrast, Jurkat is a clone, and thus, there is no diversity to choose from when $\mathrm{KD}$ is performed, which would explain the crippling effect of CD3 $\gamma \mathrm{KD}$ on TCR expression. Other cell lines and primary $\mathrm{T}$ cells showed similar results (Figure 7). Primary $\mathrm{T}$ cells, although polyclonal, do not have TCR-dependent selection mechanisms as immature $\mathrm{T}$ cells do. To test our hypothesis in polyclonal cells, FTOC CD3D KD experiments were performed, in which the few $\mathrm{T}$ cells (pre-T, $\alpha \beta$, or $\gamma \delta$ ) that did emerge from the cultures expressed comparable TCR ensemble levels (Figure 9). These results support the hypothesis of intrathymic TCR expression plasticity of emerging polyclonal T lymphocytes, which can then signal for survival in $\mathrm{CD} 3 \gamma$, but not in CD3 $\delta$ deficiency and explain the immunological and clinical disparities of these ID cases.

In conclusion, our results show that both $\mathrm{CD} 3 \gamma$ and $\mathrm{CD} 3 \delta$ are required for surface TCR expression in human mature $\mathrm{T}$ cells. Our co-IP experiments show that nascent TCRs begin to form but are unable to incorporate $\zeta \zeta / \mathrm{CD} 247_{2}$ dimers and are retained in the ER. In contrast, the FTOC experiments show that the lack of those chains early in development, acting on emerging polyclonal repertoires, allows for some TCR expression plasticity

\section{REFERENCES}

Abraham, R. T., and Weiss, A. (2004). Jurkat T cells and development of the T-cell receptor signalling paradigm. Nat. Rev. Immunol. 4, 301-308. doi: 10.1038/ nri1330

Bonifacino, J. S., Suzuki, C. K., and Klausner, R. D. (1990). A peptide sequence confers retention and rapid degradation in the endoplasmic reticulum. Science 247, 79-82. doi: 10.1126/science.2294595 and further T-cell development when signaling from incomplete TCRs is feasible, as is the case in CD3 $\gamma$, but not in CD3 $\delta$, ID.

Our findings could give rise to potential future therapeutic advances, including the transient reduction of $\operatorname{CD} 3 \delta$ or $\operatorname{CD} 3 \gamma$ expression by KD of mature T cells as a novel immunosuppressive therapy, particularly in those diseases with overactive T cells, such as autoimmune or alloimmune disorders.

\section{DATA AVAILABILITY STATEMENT}

The original contributions presented in the study are included in the article/supplementary material, further inquiries can be directed to the corresponding author/s.

\section{ETHICS STATEMENT}

The studies involving human participants were reviewed and approved by Comité Ético de Investigación Clínica del Hospital Universitario 12 de Octubre de Madrid. Written informed consent to participate in this study was provided by the participants' legal guardian/next of kin.

\section{AUTHOR CONTRIBUTIONS}

BG, PF, MT, and JR conceived the experimental study. BG designed and performed most of the experiments, and analyzed the data. PF did the FTOC studies (Figure 9) under the supervision of MT. RM, DC-A, and RL did TCR comparative experiments in cell lines and fresh human $\mathrm{T}$ cells (Figure 7) under the supervision of PC. MM, MM-R, and AJ-R contributed with substantial technical help to several figures. EF-M and AM contributed substantially to the manuscript revision and figures' elaboration. JR and BG wrote the manuscript. All authors participated in the manuscript revision before submission.

\section{FUNDING}

This work was supported by grants from the Ministerio de Economía y Competitividad (MINECO RTI2018-095673-B-I00 and PID2019-105623RB-I00), Comunidad Autónoma de Madrid (CAM B2017/BMD3673), and Asociación Española Contra el Cáncer (AECC PROYE20084REGU and CICPF18030TORI), Fundación Ramón Areces, and Fundación Unoentrecienmil. AM was supported by Complutense/Harvard University scholarship CT46/15 and AJ-R by MINECO scholarship BES-2012-055054. 
the endoplasmic reticulum. EMBO J. 23, 2348-2357. doi: 10.1038/sj.emboj. 7600245

Dadi, H. K., Simon, A. J., and Roifman, C. M. (2003). Effect of CD3delta deficiency on maturation of alpha/beta and gamma/delta T-cell lineages in severe combined immunodeficiency. N. Engl. J. Med. 349, 1821-1828. doi: 10.1056/NEJMoa031178

Dave, V. P., Cao, Z., Browne, C., Alarcon, B., Fernandez-Miguel, G., Lafaille, J., et al. (1997). CD3 delta deficiency arrests development of the alpha beta but not the gamma delta T cell lineage. EMBO J. 16, 1360-1370. doi: 10.1093/emboj/16. 6.1360

Delgado, P., and Alarcon, B. (2005). An orderly inactivation of intracellular retention signals controls surface expression of the $\mathrm{T}$ cell antigen receptor. J. Exp. Med. 201, 555-566. doi: 10.1084/jem.20041133

Dietrich, J., Kastrup, J., Lauritsen, J. P., Menne, C., von Bulow, F., and Geisler, C. (1999). TCRzeta is transported to and retained in the Golgi apparatus independently of other TCR chains: implications for TCR assembly. Eur. J. Immunol. 29, 1719-1728.

Garaulet, G., Alfranca, A., Torrente, M., Escolano, A., López-Fontal, R., Hortelano, S., et al. (2013). IL10 released by a new inflammation-regulated lentiviral system efficiently attenuates zymosan-induced arthritis. Mol. Ther. 21, 119-130.

García-Peydró, M., de Yébenes, V. G., and Toribio, M. L. (2003). Sustained Notch1 signaling instructs the earliest human intrathymic precursors to adopt a gammadelta T-cell fate in fetal thymus organ culture. Blood 102, 2444-2451.

Garcillán, B., Mazariegos, M. S., Fisch, P., Res, P. C., Muñoz-Ruiz, M., Gil, J., et al. (2014). Enrichment of the rare CD $4+\gamma \delta$ T-cell subset in patients with atypical CD3 $\delta$ deficiency. J. Allergy Clin. Immunol. 133, 1205-1208. doi: 10.1016/j.jaci. 2013.10.002

Geisler, C. (1992). Failure to synthesize the CD3-gamma chain. Consequences for $\mathrm{T}$ cell antigen receptor assembly, processing, and expression. J. Immunol. 148, 2437-2445.

Gil, J., Busto, E. M., Garcillán, B., Chean, C., García-Rodríguez, M. C., DíazAlderete, A., et al. (2011). A leaky mutation in CD3D differentially affects $\alpha \beta$ and $\gamma \delta \mathrm{T}$ cells and leads to a $\mathrm{T} \alpha \beta-\mathrm{T} \gamma \delta+\mathrm{B}+\mathrm{NK}+$ human SCID. J. Clin. Invest. 121, 3872-3876. doi: 10.1172/jci44254

Göbel, T. W., and Dangy, J. P. (2000). Evidence for a stepwise evolution of the CD3 family. J. Immunol. 164, 879-883. doi: 10.4049/jimmunol.164.2.879

Haks, M. C., Krimpenfort, P., Borst, J., and Kruisbeek, A. M. (1998). The CD3gamma chain is essential for development of both the TCRalphabeta and TCRgammadelta lineages. EMBO J. 17, 1871-1882. doi: 10.1093/emboj/17.7. 1871

Hewitt, C. R., Lamb, J. R., Hayball, J., Hill, M., Owen, M. J., and O’Hehir, R. E. (1992). Major histocompatibility complex independent clonal T cell anergy by direct interaction of Staphylococcus aureus enterotoxin B with the T cell antigen receptor. J. Exp. Med. 175, 1493-1499. doi: 10.1084/jem.175.6.1493

Kappes, D. J., and Tonegawa, S. (1991). Surface expression of alternative forms of the TCR/CD3 complex. Proc. Natl. Acad. Sci. U.S.A. 88, 1061910623.

Lee, S. J. (1998). Endoplasmic reticulum retention and degradation of T cell antigen receptor beta chain. Exp. Mol. Med. 30, 159-164. doi: 10.1038/emm.1998.23

Letourneur, F., and Klausner, R. D. (1992). A novel di-leucine motif and a tyrosinebased motif independently mediate lysosomal targeting and endocytosis of CD3 chains. Cell 69, 1143-1157. doi: 10.1016/0092-8674(92)90636-q

Love, P. E., and Hayes, S. M. (2010). ITAM-mediated signaling by the T-cell antigen receptor. Cold Spring Harb. Perspect. Biol. 2:a002485. doi: 10.1101/cshperspect. a002485
Marin, A. V. M., Garcillán, B., Jiménez-Reinoso, A., Muñoz-Ruiz, M., Briones, A. C., Fernández-Malavé, E., et al. (2015). Human congenital T-cell receptor disorders. Lymphosign J. 2, 3-19. doi: 10.14785/lpsn-2014-0012

Pacheco-Castro, A., Alvarez-Zapata, D., Serrano-Torres, P., and Regueiro, J. R. (1998). Signaling through a CD3 gamma-deficient TCR/CD3 complex in immortalized mature CD4+ and CD8+ T lymphocytes. J. Immunol. 161, 31523160 .

Perez-Aciego, P., Alarcon, B., Arnaiz-Villena, A., Terhorst, C., Timon, M., Segurado, O. G., et al. (1991). Expression and Function of a Variant T Cell Receptor Complex Lacking CD3g. J. Exp. Med. 174, 319-326.

Rowe, J. H., Delmonte, O. M., Keles, S., Stadinski, B. D., Dobbs, A. K., Henderson, L. A., et al. (2018). Patients with CD3G mutations reveal a role for human CD3 $\gamma$ in Treg diversity and suppressive function. Blood 131, 2335-2344. doi: 10.1182/blood-2018-02-835561

Schamel, W. W., Alarcon, B., and Minguet, S. (2019). The TCR is an allosterically regulated macromolecular machinery changing its conformation while working. Immunol. Rev. 291, 8-25. doi: 10.1111/imr.12788

Schneider, U., Schwenk, H. U., and Bornkamm, G. (1977). Characterization of EBV-genome negative "null" and "T" cell lines derived from children with acute lymphoblastic leukemia and leukemic transformed non-Hodgkin lymphoma. Int. J. Cancer 19, 621-626. doi: 10.1002/ijc.2910190505

Szymczak, A. L., Workman, C. J., Wang, Y., Vignali, K. M., Dilioglou, S., Vanin, E. F., et al. (2004). Correction of multi-gene deficiency in vivo using a single 'self-cleaving' 2A peptide-based retroviral vector. Nat. Biotechnol. 22, 589-594. doi: $10.1038 / \mathrm{nbt} 957$

Wegener, A. M., Hou, X., Dietrich, J., and Geisler, C. (1995). Distinct domains of the CD3-gamma chain are involved in surface expression and function of the T cell antigen receptor. J. Biol. Chem. 270, 4675-4680. doi: 10.1074/jbc.270.9. 4675

Wileman, T., Carson, G. R., Concino, M., Ahmed, A., and Terhorst, C. (1990). The gamma and epsilon subunits of the CD3 complex inhibit pre-Golgi degradation of newly synthesized T cell antigen receptors. J. Cell Biol. 110, 973-986. doi: $10.1083 /$ jcb.110.4.973

Wileman, T., Kane, L. P., Young, J., Carson, G. R., and Terhorst, C. (1993). Associations between subunit ectodomains promote $\mathrm{T}$ cell antigen receptor assembly and protect against degradation in the ER. J. Cell Biol. 122, 67-78. doi: 10.1083/jcb.12 2.1.67

Wucherpfennig, K. W., Gagnon, E., Call, M. J., Huseby, E. S., and Call, M. E. (2010). Structural biology of the T-cell receptor: insights into receptor assembly, ligand recognition, and initiation of signaling. Cold Spring Harb. Perspect. Biol. 2:a005140. doi: 10.1101/cshperspect.a005140

Conflict of Interest: The authors declare that the research was conducted in the absence of any commercial or financial relationships that could be construed as a potential conflict of interest.

Copyright (C) 2021 Garcillán, Fuentes, Marin, Megino, Chacon-Arguedas, Mazariegos, Jiménez-Reinoso, Muñoz-Ruiz, Laborda, Cárdenas, FernándezMalave, Toribio and Regueiro. This is an open-access article distributed under the terms of the Creative Commons Attribution License (CC BY). The use, distribution or reproduction in other forums is permitted, provided the original author(s) and the copyright owner(s) are credited and that the original publication in this journal is cited, in accordance with accepted academic practice. No use, distribution or reproduction is permitted which does not comply with these terms. 